\title{
Interrelationship of RNA Interference and Transcriptional Gene Silencing in Drosophila
}

\author{
M. PAL-BHADRA, $\stackrel{* \ddagger}{\ddagger}$ U. BHADRA, ${ }^{\dagger \dagger}$ AND J.A. BIRCHLER \\ *Division of Biological Sciences, University of Missouri-Columbia, Columbia, Missouri 65211
}

Gene silencing processes that operate within the nucleus first came to light with studies of transgene silencing in plants (Matzke et al. 1989; Napoli et al. 1990; van der Krol et al. 1990). This body of work suggested the involvement of double-stranded RNA molecules as the catalyst for posttranscriptional silencing (Metzlaff et al. 1997). Studies of plant viruses, which are targets of posttranscriptional silencing (Goodwin et al. 1996), resulted in the finding of RNA-dependent DNA methylation in the nucleus (Wassenegger et al. 1994; Mette et al. 1999, 2000; Jones et al. 2001). When the finding was made that the experimental procedure of RNAi likely worked through a double-stranded RNA process (Fire et al. 1998), biochemical studies were inspired that led to the discovery of what is referred to as the "RNAi machinery" (Tuschl et al. 1999; Hammond et al. 2000, 2001; Zamore et al. 2000; Bernstein et al. 2001). This machinery not only works to destroy homologous messenger RNAs in a posttranscriptional manner (Montgomery et al. 1998), using siRNAs as a guide (Hamilton and Baulcombe 1999), but also is involved with processing of micro-RNAs that play a role in normal development of plants and animals (Grishok et al. 2001; Hutvagner et al. 2001) as well as with targeting sites on the chromosomes for chromatin modifications, which ultimately result in transcriptional silencing (Pal-Bhadra et al. 1997, 2002, 2004; Morel et al. 2000; Sijen et al. 2001; Hall et al. 2002; Volpe et al. 2002; Schramke and Allshire 2003; Verdel et al. 2004). In this paper we will focus on studies in our laboratory concerned with novel aspects of transcriptional gene silencing and its interrelationship with the RNAi machinery.

These studies began with the construction of a promoter-reporter fusion transformant designed to study regulatory factors effective on the white eye color gene in Drosophila (Rabinow et al. 1991). This plasmid consists of the white gene promoter fused to the Alcohol dehydrogenase structural gene in the respective 5' leader sequences. It was transformed into a background carrying an $A d h$ allele with very low expression. This $w$-Adh transgene was expressed less when homozygous at any location in the genome (Rabinow et al. 1991) than when only

\footnotetext{
*Present address: Department of Pharmacology, Indian Institute of Chemical Biology, Hyderabad 500007, India.

${ }^{\dagger}$ Present address: Functional Genomics and Gene Silencing Group, Centre for Cellular and Molecular Biology, Hyderabad 500007, India.

¥These authors contributed equally.
}

a single copy was present. This behavior was reminiscent of the phenomenon of pairing-sensitive silencing described by Judy Kassis for engrailed-white transgenes (Kassis et al. 1991; Kassis 1994; Americo et al. 2002).

A further unusual property of the $w$-Adh transgenes was that they were not additive in expression when multiple copies were present in the cell at dispersed locations (Pal-Bhadra et al. 1997). This type of behavior was analogous to the phenomenon of cosuppression that had been described in plant species for numerous transgenes. This silencing was modulated by various mutations in the genes encoding the Polycomb complex of repressive chromatin proteins. Indeed, when silencing is operative, the silenced transgenes become ectopically associated with the Polycomb complex. This type of silencing occurs at the transcriptional level (Pal-Bhadra et al. 2002).

Other transgenes, such as a full-length $A d h$ construct, exhibit silencing that operates at the posttranscriptional level (Pal-Bhadra et al. 2002). This type of silencing is associated with the production of siRNAs and thus fulfills all the hallmarks of operating via the RNA interference pathway. Mutations in this type of silencing are blocked by mutations in the piwi gene (Cox et al. 1998), which is a member of a gene family characterized by the PAZ domain. Members of this family are involved with RNAi functions in many species (Cerutti et al. 2000).

The transcriptional silencing characteristic of an interaction between $w$-Adh and a reciprocal Adh-white transgene is also blocked by mutation in the piwi gene (PalBhadra et al. 1999, 2002) and to a lesser extent by mutations in another member of the same gene family, aubergine (Schupbach and Wieschaus 1991; Schmidt et al. 1999; Birchler et al. 2003). These findings indicated a connection between posttranscriptional and transcriptional silencing in Drosophila.

With the finding that heterochromatic silencing in fission yeast was suppressed by mutations in the RNAi machinery (Hall et al. 2002; Volpe et al. 2002), tests were conducted as to whether such mutations in Drosophila would suppress various types of heterochromatin-based silencing (Pal-Bhadra et al. 2004). Both piwi and aubergine suppress mini-white insertions in the fourth chromosome heterochromatin. Another gene, homeless, which encodes a double-stranded RNA helicase required for RNAi (Aravin et al. 2001; Stapleton et al. 2001; Kennerdell et al. 2002), suppresses these transgene insertions as well as repeat-induced silencing of a tandem array of 
miniwhite (Dorer and Henikoff 1994), which shows variegated silencing. These findings provide further evidence of an involvement of the RNAi machinery in the establishment of chromatin domains.

\section{IS THERE A RELATIONSHIP BETWEEN COSUPPRESSION AND PAIRING-SENSITIVE SILENCING?}

The trans silencing of $w$-Adh and involvement of the Polycomb complex raises the issue of whether an analogous mechanism operates on the silencing observed with transgenes that carry a Polycomb Response Element (PRE). These transgenes typically exhibit pairing-sensitive silencing, as does $w$-Adh. In those cases examined, increased copy number in the nucleus results in further decline in total expression of the collective homologous transgenes (Hagstrom et al. 1997; Sigrist and Pirrotta 1997; Muller et al. 1999), again in analogy with $w$-Adh.

The engrailed-white transgenes carry a PRE and were the prototypical case of pairing-sensitive silencing (Kassis et al. 1991; Kassis 1994; Brown et al. 1998; Americo et al. 2002). We used multiple dispersed copies to test whether dispersed silencing was also operative with this transgene. When a large fragment of engrailed (2595 bp) was carried on the transgene adjacent to the mini-white reporter insertions at various locations in the genome, they exhibited the normal pairing-sensitive silencing. The general assay for pairing-sensitive silencing is a test of whether two paired copies are expressed less than two copies unpaired in the genome. This is indeed the case for insertions of this class (the G series). An example is shown in Figure 1. However, two unpaired copies are not additive in their expression, and further increase in copy

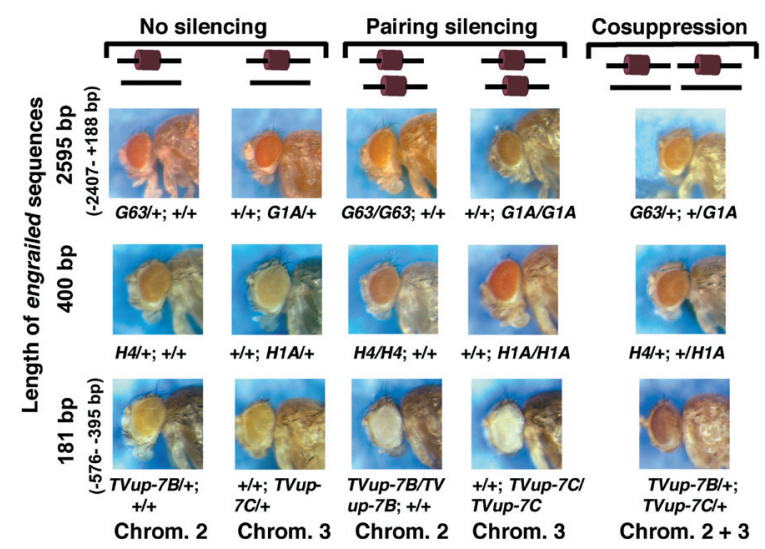

Figure 1. The eye color of multiple engrailed-white (en-w) transgenes showing a comparison of pairing-sensitive silencing and cosuppression. The length of engrailed sequences, chromosomal location, and allelic combinations are noted in the panel. Each en-w construct was tested in at least two genomic locations. The engrailed-white gene carrying approximately $2595 \mathrm{bp}$ of engrailed sequences shows pairing-sensitive silencing. The presence of $400 \mathrm{bp}$ of engrailed sequences next to mini-white shows a gene dosage effect in paired copies at each location. When only 181 bp upstream to the 400-bp sequence are present, pairing-sensitive silencing occurs, but cosuppression of dispersed copies is not observed.

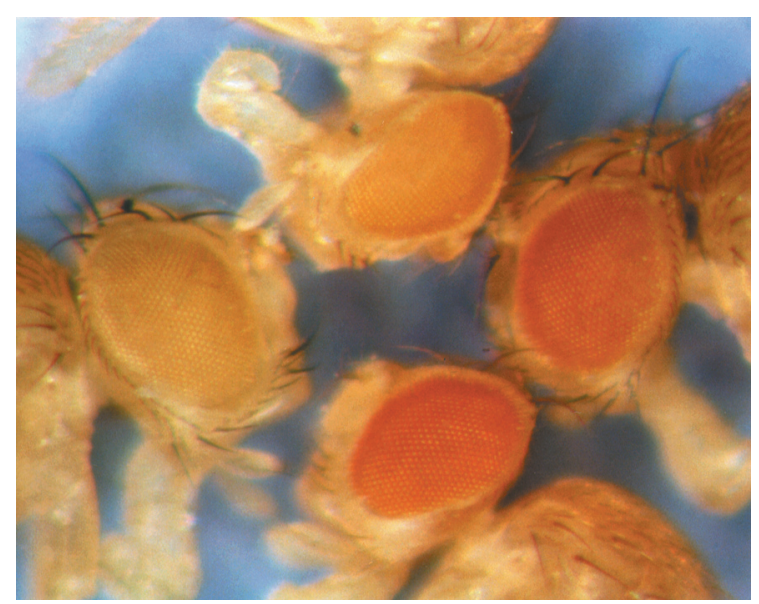

Figure 2. One to four doses of the en-w (series G) transgene shows declining total expression. At the bottom is one copy of en-w. Counterclockwise are two copies, each unpaired; three copies consisting of two copies paired with each other and a third copy unpaired; four copies in which two insert sites are both homozygous. Although not shown, the four copies (two insertion sites homozygous) are expressed less than either paired insert alone.

number causes less total expression (Fig. 2). The pairing effects and trans-acting silencing are also reflected in the RNA level of white expression (Fig. 3). This departure from additivity illustrates that this class of $e n-w$ transgene participates in dispersed transgene silencing as well, a phenomenon referred to as cosuppression.

A second class of en-w transgenes (the $\mathrm{H}$ series) has only $400 \mathrm{bp}$ of engrailed adjacent to the mini-white reporter. In this case the sequences of engrailed thought to be the major determinant of pairing-sensitive silencing are missing. When the transgenes are paired at homologous sites, the expression of the mini-white reporter is additive in the homozygotes for the respective insertion sites (Figs. 1 and 4). With respect to dispersed silencing, there is no cosuppression observed with this particular transgene (Figs. 1 and 4).

Last, a transgene containing only the portion of engrailed thought to play a major role in attracting the Polycomb complex was tested. This transgene series (TVup) contained only 181 base pairs of the engrailed gene. This small segment is sufficient to condition pairing-sensitive silencing (Figs. 1 and 5). Interestingly, however, this transgene construct does not exhibit dispersed transgene silencing. Therefore, although there may be determinants in common between pairing-sensitive silencing and cosuppression, the results with this series suggest that those sequences that can confer pairing-sensitive silencing are insufficient to produce cosuppression or that some sequence in this fragment can interfere with cosuppression.

\section{MUTATIONS IN THE RNAi MACHINERY AFFECT PAIRING-SENSITIVE SILENCING}

Given that the Polycomb complex has been implicated in both cosuppression and pairing-sensitive silencing, albeit with mechanistic distinctions, it was of interest to test 


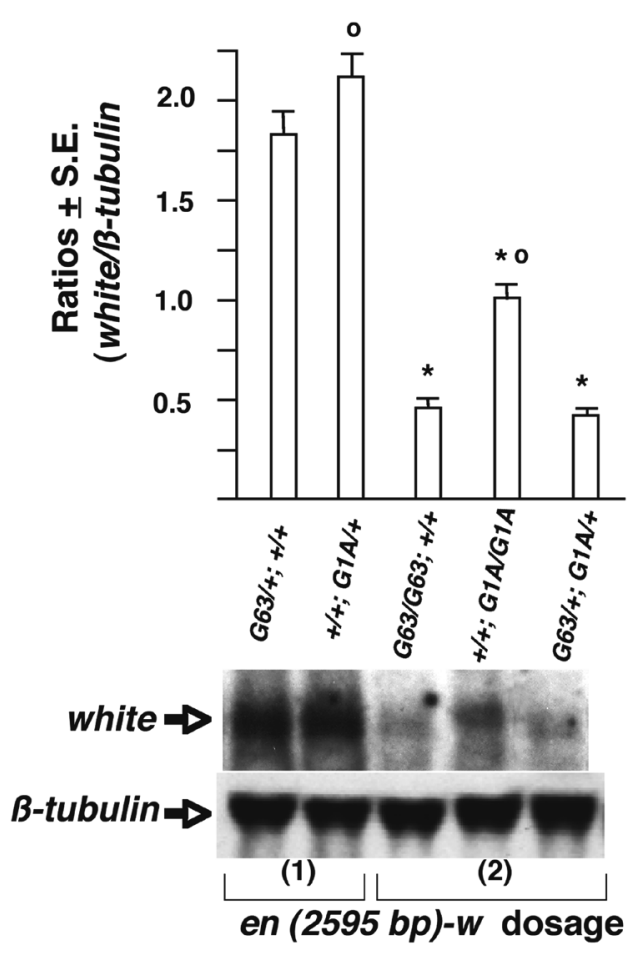

Figure 3. Autoradiograms of Northern blot hybridization from adult female flies carrying 1 and 2 copies of en-w constructs demonstrate the trends of interaction at the transcript level. Although only blots using female RNA are shown, males and females follow a similar pattern. The genotypes depicted in the autoradiogram are as follows: (1) en-w\#2/+ (chromosome 2), (2) +/en-w\#3 (chromosome 3), (3) en-w\#2/enw\#2, (4) en$w \# 3 / e n-w \# 3$, and (5) en-w\#2/en-w\#3. The bottom panel represents $\beta$-tubulin transcript level after reprobing the same blot, which acts as a gel loading control. The abundance of white transcript relative to $\beta$-tubulin loading from three independent blots is presented by a bar diagram above the panels. The error bars delimit the $95 \%$ confidence intervals. Those values significantly different between the two inserts are designated with an open circle. Those significantly different between the paired and unpaired condition are designated with an asterisk. All flies are in a $y w[67 c 23]$ background. The autoradiogram shows a reduction of white transcripts for the paired copies relative to the single insert at each location. Note that both pairing silencing and cosuppression are reflected in the level of the white transcripts.

whether pairing-sensitive silencing would be modulated by mutations in the RNAi machinery as is cosuppression. Thus, various insertions of the engrailed-white transgene were combined with different alleles of piwi and homeless.

The insertions A2 and G1A, both of which exhibit pairing-sensitive silencing, were genetically combined with $p i w i^{1}, p i w i^{2}$, and the heteroallelic combination. The homozygous piwi mutations do not have any discernible phenotypic effect on a single unpaired copy of either insertion. However, the paired copies have reduced expression, which is diminished even further in the mutant piwi homozygotes. In other words, the silencing is greater in the absence of the functional piwi gene product.

Similarly, the combination of en-w 1-1B construct, which resides on the $\mathrm{X}$ chromosomes and exhibits pair-

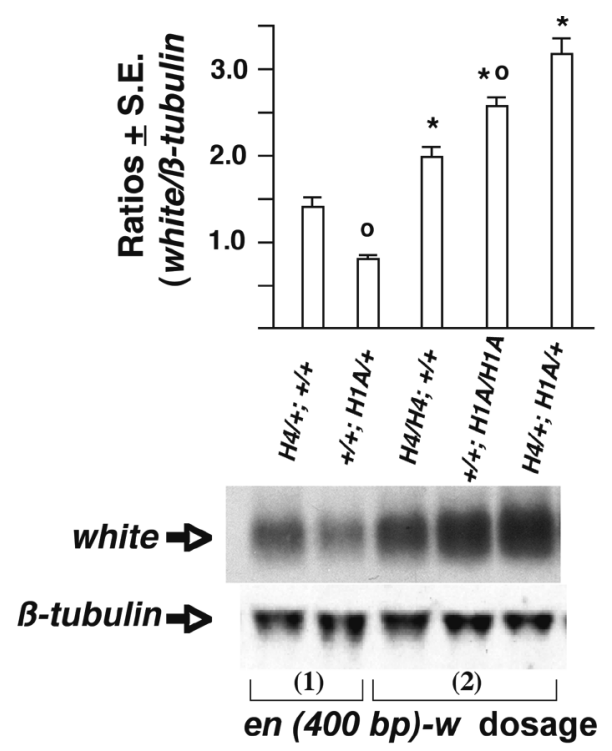

Figure 4. The white transcripts of truncated (400-bp) en-w inserts are proportionately increased with the copy number, suggesting a gene dosage effect rather than pairing-sensitive silencing and cosuppression. Copy number of the representative transgenes is arranged as described in Fig. 3.

ing-sensitive silencing, with homeless alleles produced a related result. The alleles of homeless tested were $D E 8$ and delta125, as well as their heteroallelic combination. Again, the homeless alleles had no apparent phenotypic effect on an unpaired en-w transgene when homozygous, but cause a greater silencing of the paired situation. The magnitude of enhancement of pairing-sensitive silencing

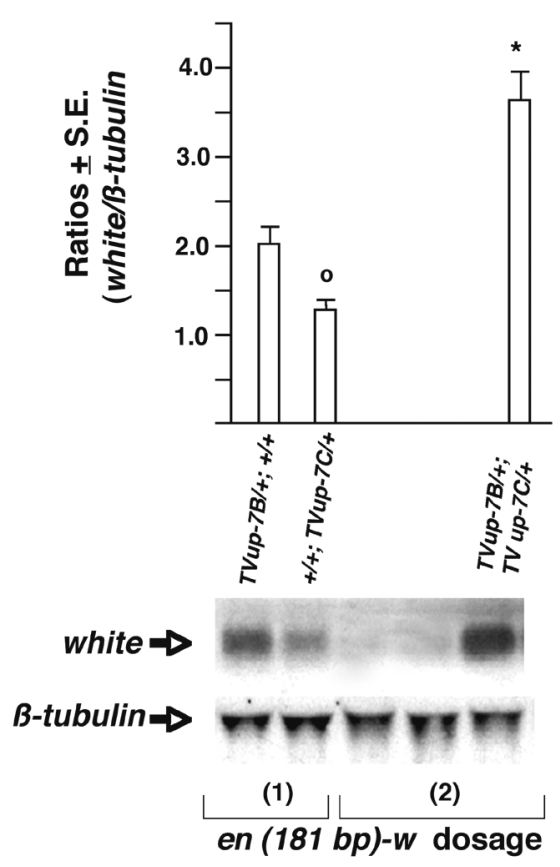

Figure 5. The TVup series exhibits pairing-sensitive silencing, but not cosuppression. Copy number of the representative transgenes is arranged as described in Fig. 3. 


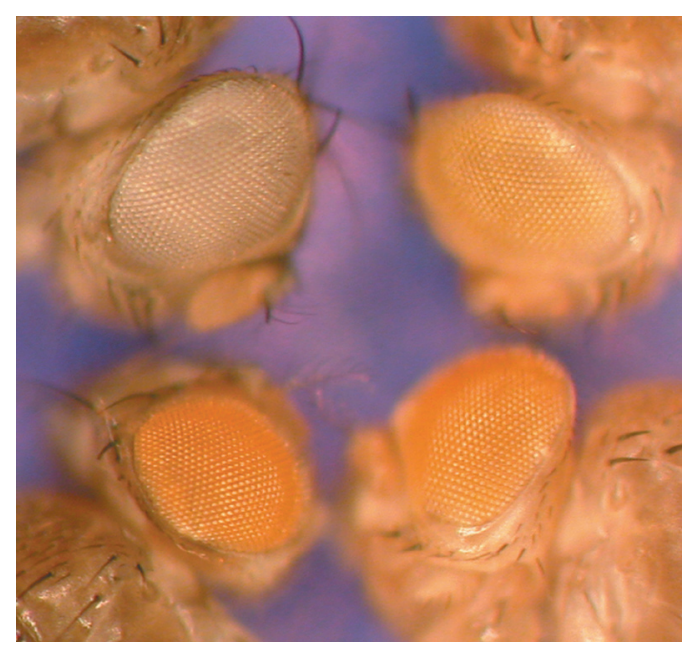

Figure 6. Pairing-sensitive silencing is enhanced by homeless mutations. The 1-1B engrailed-white construct on the $\mathrm{X}$ chromosome was combined with the homeless alleles, delta125 and $D E 8$, as well as their heteroallelic combination. Similar results were found in all cases. Depicted are flies segregating for the delta125 allele. Bottom right, a male carrying a single copy of the en-w transgene. Top right, a female homozygous for this construct, which exhibits pairing-sensitive silencing. Bottom left, a male with an unpaired en-w insertion and with the homeless mutation homozygous. Top left, a female homozygous for the transgene and for homeless. Note that homeless has little effect on the unpaired transgene, but conditions a stronger silencing of $e n-w$ when it is paired.

by homeless was greater than with piwi. The effect of homeless on pairing-sensitive silencing is shown in Figure 6.

\section{CONCLUSIONS}

Several lines of investigations indicate that the RNAi machinery operates via a posttranscriptional mechanism to destroy specific messenger RNAs or viruses. The trigger for these processes appears to be the production of a double-stranded RNA moiety. One natural function is likely to be a defense mechanism against the overexpression of transposable elements (Chabossier et al. 1998; Jensen et al. 1999; Ketting et al. 1999; Tabara et al. 1999) and against viruses. Transposable elements are present at many sites in the chromosomes and can therefore be transcribed in various orientations in such a manner as to contribute to a pool of aberrant RNAs that will trigger such a response. The double-stranded RNAs are cleaved to siRNAs, which are incorporated into the RISC complex to serve as a guide for the enzymatic destruction of the homologous RNAs.

Transcriptional silencing is less well understood but requires functional gene products of many of the same components as posttranscriptional RNA interference (PalBhadra et al. 2002, 2004). Transgene silencing accompanied by the accumulation of the Polycomb complex must involve homology recognition of the related sequences throughout the nucleus for the establishment of this silencing complex. The most likely candidate for this trans-acting function are small RNAs, although how they are generated and function at the chromosomal level is still unknown.

Transcriptional silencing of heterochromatic repeats in Schizosaccharomyces pombe (Hall et al. 2002; Volpe et al. 2002) and Drosophila (Pal-Bhadra et al. 2004) also require the functional gene products of the RNAi machinery. In this case, it is postulated that the repeats generate small RNAs that in turn target chromatin modifications that foster silencing, namely, histone $\mathrm{H} 3$ methylated at lysine 9 (H3-mK9). This modified form of histone H3 is bound by Swi6 in fission yeast and by Heterochromatin Protein 1 (HP1) in Drosophila. Thus, in this type of transcriptional silencing as well, siRNAs are implicated in guiding the chromatin modification machinery to homologous sequences in the nucleus to silence the various copies of repeated sequences.

An additional type of transcriptional silencing involves the contact or pairing of gene sequences. Transfer of a silenced state from one allele to another was documented during meiosis in Ascobolus (Colot et al. 1996). The silencing followed the same parameters as gene conversion, suggesting similarities between homology recognition for silencing and recombination. In Neurospora, also during meiosis, silencing of repeated sequences within the nucleus is triggered by the presence of an unpaired sequence (Shiu et al. 2001; Shiu and Metzenberg 2002). Such unpaired DNA will cause the silencing of all homologous copies whether paired or not. This process is referred to as meiotic silencing by unpaired DNA (MSUD). Interestingly, it is suppressed by mutations in the RNAi machinery (Shiu et al. 2001; Shiu and Metzenberg 2002; Lee et al. 2003).

As noted above, a pairing-dependent silencing phenomenon occurs in Drosophila and is referred to as pairing-sensitive silencing. It is typically manifested in the behavior of transgenes that express well as one copy, but less well when the transgene is homozygous. In this case, pairing of the transgenes can occur somatically given the property of Drosophila homologs to associate in all tissues.

We investigated the relationship of pairing-sensitive silencing to cosuppression and the RNAi machinery in the experiments described above. The larger engrailed-white transgenes participate in cosuppression in that they exhibit nonlinearity of expression relative to the copy number present in the nucleus.

Mutations in the RNAi machinery genes, piwi and homeless, were tested for an impact on pairing-sensitive silencing. Interestingly, they both increase the degree of silencing rather than suppress the loss of expression, raising interesting issues about how the RNAi machinery is involved in a process that appears to involve gene-togene contact.

One possibility might be that the paired versus unpaired configuration impacts the nature of gene transcription and modifies the production of messenger RNAs that are abnormal. Abnormal RNAs are thought to enter into the RNAi pathway to eventually generate siRNAs, which 
may in turn feed back to the gene to modify the local chromatin environment.

A second possibility is simply that the RNAi machinery is intimately associated with the transgenes and acts to inhibit the silencing that might otherwise occur as a result of gene-to-gene contact. When the machinery is lost in the mutants, the silencing can be more severe.

A third possibility is that the gene products of the RNAi machinery are shared with other processes. The RNAi machinery must use the siRNAs as a homology guide for targeting the destruction of mRNAs in the cytoplasm as well as for guiding the sites of chromatin modifications in the nucleus. Pairing-sensitive silencing likely involves homology recognition in some form, followed by a change of chromatin state. It is possible, but not known at present, whether protein components of the RNAi machinery are utilized in other molecular complexes that perform related functions, such as DNA homology recognition.

A fourth possibility is that siRNAs might also be used as a guide to establish chromatin modifications in domains that foster gene activation, as well as those that foster gene silencing. Most research to date implicates siRNAs and the RNAi machinery as the responsible agent for determining the sites of chromatin modifications in the nucleus that result in silencing of all homologous sequences. If, however, the RNAi machinery helps maintain the activity of genes by related means, then the mutational inactivation of the machinery would allow greater silencing to occur.

Polycomb-dependent silencing and heterochromatic silencing are suppressed by mutations in the RNAi machinery. Here we provide evidence that components of this cellular process also affect gene expression as impacted by gene associations. Thus, there is increasing evidence that the RNAi machinery plays an important role in establishing the chromatin configurations along the length of the chromosome.

\section{ACKNOWLEDGMENTS}

We thank Judy Kassis for providing en-w transgenes and for discussions. Research was supported by a grant from the National Science Foundation MCB 0211376.

\section{REFERENCES}

Americo J., Whitely M., Brown J.L., Fujioka M., Jaynes J.B., and Kassis J.A. 2002. A complex array of DNA-binding proteins required for pairing-sensitive silencing by a polycomb group response element from the Drosophila engrailed gene. Genetics 160: 1561.

Aravin A.A., Naumova N.M., Tulin A.A., Rozovsky Y.M., and Gvozdev V.A. 2001. Double stranded RNA-mediated silencing of genomic tandem repeats and transposable elements in Drosophila melanogaster germline. Curr. Biol.11: 1017.

Bernstein E., Caudy A.A., Hammond S.M., and Hannon G.J. 2001. Role for a bidentate ribonuclease in the initiation step of RNA interference. Nature 409: 295.

Birchler J.A., Pal-Bhadra M., and Bhadra U. 2003. Transgene cosuppression in animals. In RNAi: A guide to gene silencing (ed. G.J. Hannon), p. 23. Cold Spring Harbor Laboratory Press, Cold Spring Harbor, New York.
Brown J.L., Mucci D., Whiteley M., Dirksen M.-L., and Kassis J.A. 1998. The Drosophila Polycomb group gene pleiohomeotic encodes a DNA binding protein with homology to the transcription factor YY1. Mol. Cell 1: 1057.

Cerutti L., Mian N., and Bateman A. 2000. Domains in gene silencing and cell differentiation proteins: The novel PAZ domain and redefinition of the piwi domain. Trends Biochem. Sci. 25: 481 .

Chabossier M.-C., Bucheton A., and Finnegan D.J. 1998. Copy number control of a transposable element, the I factor, a LINE-like element in Drosophila. Proc. Natl. Acad. Sci. 95: 11781.

Colot V., Maloisel L., and Rossignol J.L. 1996. Interchromosomal transfer of epigenetic states in Ascobolus: Transfer of DNA methylation is mechanistically related to homologous recombination. Cell 86: 855.

Cox D.N., Chao A., Baker J., Chang L., Qiao D., and Lin H. 1998. A novel class of evolutionary conserved genes defined by piwi are essential for stem cell self-renewal. Genes Dev. 12: 3715

Dorer D.R. and Henikoff S. 1994. Expansions of transgene repeats cause heterochromatin formation and gene silencing in Drosophila. Cell 77: 993.

Fire A., Xu S., Montgomery M.K., Kostas S.A., Driver S.E., and Mello C.C. 1998. Potent and specific genetic interference by double-stranded RNA in Caenorhabditis elegans. Nature 391: 806.

Goodwin J., Chapman K., Swaney S., Parks T.D., Wernsman E.A., and Doughtery W.G. 1996. Genetic and biochemical dissection of transgenic RNA-mediated virus resistance. Plant Cell 8: 95.

Grishok A., Pasquinelli A.E., Conte D., Li N., Parrish S., Ha I., Baillie D.L., Fire A., Ruvkun G., and Mello C.C. 2001. Genes and mechanisms related to RNA interference regulate expression of the small temporal RNAs that control C. elegans developmental timing. Cell 106: 23.

Hagstrom K., Muller M., and Schedl P. 1997. A Polycomb and GAGA dependent silencer adjoins the Fab-7 boundary in the Drosophila bithorax complex. Genetics 146: 1365.

Hall I.M., Shankaranarayana G.D., Noma K., Ayoub N., Cohen A., and Grewal S.I. 2002. Establishment and maintenance of a heterochromatin domain. Science 297: 2232.

Hamilton A.J. and Baulcombe D.C. 1999. A species of small antisense RNA in posttranscriptional gene silencing in plants. Science 286: 950.

Hammond S.C., Boettcher S., Caudy A.A., Kobayashi R., and Hannon G.J. 2001. Argonaute2, a link between genetic and biochemical analyses of RNAi. Science 293: 1146.

Hammond S.M., Bernstein E., Beach D., and Hannon G.J. 2000. An RNA-directed nuclease mediates post-transcriptional gene silencing in Drosophila cells. Nature 404: 293.

Hutvagner G., McLachlan J., Pasquinelli A.E., Balint E., Tuschl T., and Zamore P.D. 2001. A cellular function for the RNAinterference enzyme Dicer in the maturation of the let-7 small temporal RNA. Science 293: 834.

Jensen S., Gassama M.-P., and Heidmann T. 1999. Taming of transposable elements by homology dependent gene silencing. Nat. Genet. 21: 209.

Jones L., Ratcliff F., and Baulcombe D.C. 2001. RNA-directed transcriptional gene silencing in plants can be inherited independently of the RNA trigger and requires Metl for maintenance. Curr. Biol. 11: 747.

Kassis J.A. 1994. Unusual properties of regulatory DNA from the Drosophila engrailed gene: Three "pairing sensitive" sites within a $1.6 \mathrm{~kb}$ region. Genetics 136: 1025 .

Kassis J.A., VanSickle E.P., and Sensabaugh S.M. 1991. A fragment of engrailed regulatory DNA can mediate transvection of the white gene in Drosophila. Genetics 128: 751.

Kennerdell J.R., Yamaguchi S., and Carthew R.W. 2002. RNAi is activated during Drosophila oocyte maturation in a manner dependent on aubergine and spindle-E. Genes Dev. 16: 1884.

Ketting R.F., Haverkamp T.H., van Luenen H.G.A.M., and Plasterk H.A. 1999. mut-7 of C. elegans, required for transposon 
silencing and RNA interference, is a homolog of Werner syndrome helicase and RNaseD. Cell 99: 133.

Lee D.W., Pratt R.J., McLaughlin M., and Aramayo R. 2003. An argonaute-like protein is required for meiotic silencing. $\mathrm{Ge}$ netics 164: 821.

Matzke M.A., Primig M., Trnovsky J., and Matzke A.J.M. 1989. Reversible methylation and inactivation of marker genes in sequentially transformed tobacco plants. EMBO J. 8: 643 .

Mette M.F., van der Winder J., Matzke M.A., and Matzke A.J.M. 1999. Production of aberrant promoter transcripts contributes to methylation and silencing of unlinked homologous promoters in trans. EMBO J. 18: 241.

Mette M.F., Aufsatz W., van der Winder J., Matzke M.A., and Matzke A.J.M. 2000. Transcriptional silencing and promoter methylation triggered by double-stranded RNA. EMBO J. 19: 5194.

Metzlaff M., O’Dell M., Cluster P.D., and Flavell R.B. 1997. RNA-mediated RNA degradation and chalcone synthase A silencing in petunia. Cell 88: 845 .

Montgomery M.K., Xu S., and Fire A. 1998. RNA as a target of dsRNA-mediated genetic interference in Caenorhabditis elegans. Proc. Natl. Acad. Sci. 95: 15502.

Morel J., Mourrain P., Beclin C., and Vaucheret H. 2000. DNA methylation and chromatin structure affect transcriptional and post-transcriptional transgene silencing in Arabidopsis. Curr. Biol. 10: 1591 .

Muller M., Hagstrom K., Gyurkovics H., Pirrotta V., and Schedl P. 1999. The Mcp element from the Drosophila melanogaster bithorax complex mediates long-distance regulatory interactions. Genetics 153: 1333.

Napoli C., Lemieux C., and Jorgenson R. 1990. Introduction of a chimeric chalcone synthase gene in petunia results in reversible co-suppression of homologous genes in trans. Plant Cell 2: 279.

Pal-Bhadra M., Bhadra U., and Birchler J.A. 1997. Cosuppression in Drosophila: Gene silencing of Alcohol dehydrogenase by white-Adh transgenes is Polycomb dependent. Cell 90: 479 .

. 1999. Cosuppression of nonhomologous transgenes in Drosophila involves mutually related endogenous sequences. Cell 99: 35.

. 2002. RNAi related mechanisms affect both transcriptional and posttranscriptional transgene silencing in Drosophila. Mol. Cell 9: 315.

Pal-Bhadra M., Leibovitch B.A., Gandhi S.G., Rao M., Bhadra U., Birchler J.A., and Elgin S.C. 2004. Heterochromatic silencing and HP1 localization in Drosophila are dependent on the RNAi machinery. Science 303: 669.

Rabinow L., Nguyen-Huynh A.T., and Birchler J.A. 1991. A trans-acting regulatory gene that inversely affects the expression of the white, brown and scarlet loci in Drosophila. Genetics 129: 463.
Schmidt A., Palumbo G., Bozzetti M.P., Tritto P., Pimpinelli S., and Schafer U. 1999. Genetic and molecular characterization of sting, a gene involved in crystal formation and meiotic drive in the male germ line of Drosophila melanogaster. Genetics 151: 749.

Schramke V. and Allshire R. 2003. Hairpin RNAs and retrotransposon LTRs effect RNAi and chromatin-based gene silencing. Science 301: 1069.

Schupbach T. and Wieschaus E. 1991. Female sterile mutations on the second chromosome of Drosophila melanogaster. Genetics 129: 1119.

Shiu P.K. and Metzenberg R.L. 2002. Meiotic silencing by unpaired DNA: Properties, regulation and suppression. Genetics 161: 1483 .

Shiu P.K., Raju N.B., Zickler D., and Metzenberg R.L. 2001. Meiotic silencing by unpaired DNA. Cell 107: 905.

Sigrist C.J.A. and Pirrotta V. 1997. Chromatin insulator elements block the silencing of a target gene by the Drosophila polycomb response element (PRE) but allow trans interactions between PREs on different chromosomes. Genetics 147: 209.

Sijen T., Vijn I., Rebocho A., van Blokland R., Roelofs D., Mol J.N., and Kooter J.M. 2001. Transcriptional and posttranscriptional gene silencing are mechanistically related. Curr. Biol. 11: 436 .

Stapleton W., Das S., and McKee B.D. 2001. A role of the Drosophila homeless gene in repression of Stellate in male meiosis. Chromosoma 110: 228.

Tabara H., Sarkissian M., Kelly W.G., Fleenor J., Grishok A., Timmons L., Fire A., and Mello C.C. 1999. The rde-1 gene, RNA interference, and transposon silencing in C. elegans. Cell 99: 123 .

Tuschl R., Zamore P.D., Lehman R., Bartel D.P., and Sharp P.A. 1999. Targeted mRNA degradation by double-stranded RNA in vitro. Genes Dev. 13: 3191.

van der Krol A.R., Mur L.A., Beld M., Mol J.N.M., and Stuitje A.R. 1990. Flavonoid genes in petunia: Addition of a limited number of gene copies may lead to a suppression of gene expression. Plant Cell 2: 291.

Verdel A., Jia S., Gerber S., Sugiyama T., Gygi S., Grewal S.I.S., and Moazed D. 2004. RNAi-mediated targeting of heterochromatin by the RITS complex. Science 303: 672 .

Volpe T.A., Kidner C., Hall I.M., Teng G., Grewal S.I., and Martienssen R.A. 2002 Regulation of heterochromatic silencing and histone H3 lysine-9 mylation by RNAi. Science 297: 1833.

Wassenegger M., Heimes S., Riedel L., and Sanger H.L. 1994. RNA-directed de novo methylation of genomic sequences in plants. Cell 76: 567.

Zamore P.D., Tuschl T., Sharp P.A., and Bartel D.P. 2000. RNAi: Double-stranded RNA directs the ATP-dependent cleavage of mRNA at 21 to 23 nucleotide intervals. Cell 101: 25. 


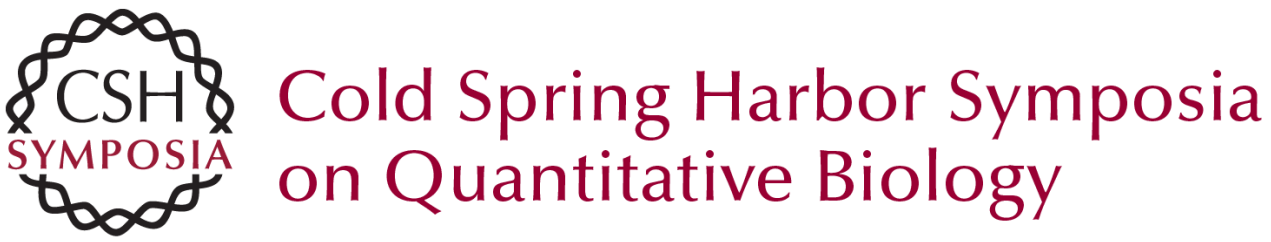

\section{Interrelationship of RNA Interference and Transcriptional Gene Silencing in Drosophila}

M. PAL-BHADRA, U. BHADRA and J.A. BIRCHLER

Cold Spring Harb Symp Quant Biol 2004 69: 433-438

Access the most recent version at doi:10.1101/sqb.2004.69.433

References This article cites 53 articles, 28 of which can be accessed free at: http://symposium.cshlp.org/content/69/433.full.html\#ref-list-1

\section{License}

Email Alerting

Receive free email alerts when new articles cite this article - sign up in Service the box at the top right corner of the article or click here. 